\title{
Egyptian sweet marjoram leaves protect against genotoxicity, immunosuppression and other complications induced by cyclophosphamide in albino rats
}

\author{
Gamal Ramadan*, Nadia M. El-Beih and Mai M. Zahra \\ Zoology Department, Faculty of Science, Ain Shams University, Abbasseya 11566 Cairo, Egypt \\ (Submitted 13 June 2011 - Final revision received 3 October 2011 - Accepted 8 October 2011 - First published online 15 December 2011)
}

\begin{abstract}
Cyclophosphamide (CP) is one of the most popular alkylating anticancer drugs that show a high therapeutic index, despite the widespread side effects and toxicity particularly in high-dose regimens and long-term use. Here, we evaluated and compared the efficacy of two different doses (50 and $100 \mathrm{mg} / \mathrm{kg}$ body weight, given orally for 30 consecutive days) of Egyptian sweet marjoram leaf powder (MLP) and marjoram leaf aqueous extract (MLE) in alleviating the genotoxicity, immunosuppression and other complications induced by CP in non-tumour-bearing albino rats. The present study showed (probably for the first time) that both MLP and MLE significantly alleviated $(P<0.05-0.001)$ most side effects and toxicity of CP-treated rats including the increase in chromosomal aberrations of bone marrow cells and serum malondialdehyde level, the decrease in the level of serum Ig, the delayed type of hypersensitivity response as also the weights and cellularity of lymphoid organs, and myelosuppression, leucopenia, macrocytic normochromic anaemia as well as thrombocytopenia by reactivating the non-enzymic (reduced glutathione) and enzymic (catalase, glutathione peroxidase, glutathione $S$-transferase, superoxide dismutase) antioxidant system and increasing the mitotic index of bone marrow cells. The modulatory effects of marjoram leaves shown in the present study were dose dependent in most cases and much higher in MLE (21-23\% for all parameters taken together). In addition, the doses used in the present study were considered safe. In conclusion, sweet marjoram leaves (especially in the form of a herbal tea) may be useful as an immunostimulant and in reducing genotoxicity in patients under chemotherapeutic interventions.
\end{abstract}

Key words: Albino rats: Clastogenicity: Cyclophosphamide: Immunosuppression: Sweet marjoram

Cyclophosphamide (CP) is an alkylating agent from the oxazaphosphorines group that is widely used to treat a variety of malignant diseases including Hodgkin's disease, nonHodgkin's lymphoma, many types of leukaemia, multiple myeloma, neuroblastomas, breast cancer, adenocarcinomas of the ovary and certain malignant neoplasms of the lung ${ }^{(1)}$. The therapeutic dose of $\mathrm{CP}$ in cancer patients is usually $100-200 \mathrm{mg} / \mathrm{kg}$ (orally and daily) or $600-1000 \mathrm{mg} / \mathrm{m}^{2}$ (intravenously every $3-4$ weeks $)^{(1)}$. Although it is an effective chemotherapeutic agent, CP intake is associated with many serious side effects and toxicity including mutagenicity, carcinogenicity, teratogenicity, myelosuppression, immunosuppression, cardiac toxicity, lung toxicity and urotoxicity, which are mainly mediated by the reactive oxygen species and lipid peroxide formation ${ }^{(2-4)}$. Also, a significant increase in micronuclei and gene mutations was found in the peripheral blood lymphocytes of nurses, pharmacists and female workers occupationally exposed to CP during its production or distribution ${ }^{(5,6)}$. Immunosuppression, particularly of humoral immunity, is a common consequence of long-term CP-chemotherapy in cancer patients ${ }^{(7,8)}$. CP-induced immunosuppression is reported to prompt various types of infection, which explains why an impressive part of the morbidity and mortality in cancer patients treated with such chemotherapeutic agents is caused by infections ${ }^{(8,9)}$. Based on the type of secondary tumours induced by $\mathrm{CP}$ in different animal species as well as in humans, it was concluded that $\mathrm{CP}$ is a systemic carcinogen (i.e. an agent that causes tumours at sites that are distal from the portal of entry, regardless of the route of exposure). This conclusion is in accordance with the assessed mutagenicity data showing that $\mathrm{CP}$ is a systemic mutagen ${ }^{(1)}$. In addition, there is evidence suggesting that $\mathrm{CP}$-associated cancers may occur in humans up to several years after drug treatment has ceased ${ }^{(1)}$. The cytotoxic side effects of

Abbreviations: CAT, catalase; CP, cyclophosphamide; GSH, reduced glutathione; GSSG, oxidised glutathione; GPx, glutathione peroxidase; GST, glutathione $S$-transferase; MDA, malondialdehyde; MLE, marjoram leaf aqueous extract; MLP, marjoram leaf powder.

*Corresponding author: Assistant Professor G. Ramadan, fax + 202 26842123, email gamal_ramadan@hotmail.com 
potentially useful chemotherapeutic agents such as CP, particularly in high-dose regimens and long-term use, on the non-target tissues are a major concern precluding their clinical use. Therefore, extensive studies have been initiated in the last decade to identify various detoxifying and protective agents that help in reducing or eliminating the undesirable side effects of $\mathrm{CP}^{(2-4,7,10-16)}$.

Sweet marjoram is one of the most popular culinary herbs in the world, which was grown in Egypt over 3000 years ago and Egypt produces $90 \%$ of the world's supply. It has also been prescribed in the form of a herbal tea (infusion) in folk medicine for asthma, cold, coughs, cramps, depression, dizziness, gastrointestinal disorders, hay fever, headache, toothache and sinus congestions, and as a diuretic and to promote menstruation $^{(17,18)}$. It contains acids (carnosic, oleanolic and ursolic acids), cis-sabinene hydrate, flavonoids (diosmetin, luteolin and apigenin), hydrocarbons (P-cymene and $\boldsymbol{\gamma}$-terpinene), phenolic glycosides (arbutin, methyl arbutin, vitexin, orientin and thymonin), phenolic terpenoids (thymol and carvacrol), tannins, sitosterol and triacontan ${ }^{(17-20)}$. According to an analysis by the US Department of Agriculture (http:// www.nal.usda.gov/fnic/cgi-bin/nut_search.pl), one teaspoon of dried marjoram leaves has $2 \mathrm{kcal}(8.4 \mathrm{KJ}), 0.36 \mathrm{~g}$ carbohydrates, $0.04 \mathrm{~g}$ fat, $0.2 \mathrm{~g}$ fibre, $0.08 \mathrm{~g}$ protein, $12 \mathrm{mg} \mathrm{Ca}$, $9 \mathrm{mg} \mathrm{K}, 0.3 \mathrm{mg}$ vitamin $\mathrm{C}, 14.4 \mu \mathrm{g}$ retinol and small amounts of other minerals and vitamins. Preliminary trials have suggested possible antioxidant properties of the sweet marjoram plant ${ }^{(20-22)}$. It was also found that the Egyptian sweet marjoram and its extracts possessed better antioxidant activities than the Hungarian ones ${ }^{(20)}$. The protective effect of sweet marjoram cultivated in Egypt against the genotoxicity induced by hydroquinone and lead acetate in mice was recently reported in two different studies ${ }^{(17,18)}$. To our knowledge, there are no reports in the literature describing the protective effects of Egyptian sweet marjoram leaves against $\mathrm{CP}$ induced genotoxicity and immunosuppression. Because marjoram leaves are commonly used as a seasoning and a tea, the present study aimed to evaluate and compare the efficacy of Egyptian sweet marjoram leaf powder (MLP) and marjoram leaf aqueous extract (MLE) in alleviating the genotoxicity, immunosuppression and other complications induced by $\mathrm{CP}$ in albino rats. Two different doses of Egyptian sweet marjoram leaves (50 and $100 \mathrm{mg} / \mathrm{kg}$ body weight) were used in the present study to test whether the obtained modulatory effects were dose dependent. Furthermore, the present study investigated any deleterious effects caused by consuming the sweet marjoram leaves.

\section{Materials and methods}

\section{Materials}

Colchicines and CP (200 mg/ampoule) were purchased from Sigma-Aldrich and Baxter Oncology $\mathrm{GmbH}$, respectively. Egyptian sweet marjoram (Origanum majorana L., family: Lamiaceae) leaves were purchased and authenticated from a herbal-specialised company (Isis Company). MLE was prepared as described previously ${ }^{(23)}$ by dissolving amounts equivalent to
50 and $100 \mathrm{mg}$ MLP/kg body weight in glassware containing $0.5 \mathrm{ml}$ boiling distilled water (equivalent to 1.5 and 3 cups of marjoram tea, respectively), then covered and let to stand for $10 \mathrm{~min}$ at room temperature. After that, the extracts were filtered and given fresh to the animals.

Adult male Wistar albino rats (Rattus norvegicus), weighing 125-135 g, were obtained from the National Research Centre in Giza, Egypt. The animals were housed in suitable cages and acclimatised to laboratory conditions for a period of 1 week before the commencement of the experiments. The rats were fed standard rodent food pellets (AgriculturalIndustrial Integration Company) and double-distilled water. The standard rodent food pellets contained wheat-bran, dried clover, maize, bean-hay, methionine, molasses, salt, in addition to minerals and vitamins mix. The amount of crude proteins, fats and fibres in the food pellets were 12, 2.4 and $14 \%$, respectively. The energy content of the standard diet was $920.48 \mathrm{~kJ} / 100 \mathrm{~g}$. All animals were humanely treated in accordance with the WHO guidelines for animal care, and the study design was approved by the Ain Shams University Research Ethics Committee.

\section{Experimental design and treatment schedule}

Experimental animals were randomly divided into ten groups of five rats each: five healthy groups; five CP-treated groups. In the healthy groups, the rats were treated by oral administration, daily with 50 or $100 \mathrm{mg} / \mathrm{kg}$ body weight (low or high dose, respectively) of either MLP suspended in $0.5 \mathrm{ml}$ distilled water (at room temperature) or MLE for $30 \mathrm{~d}$. In the CP-treated groups, non-tumour-bearing rats were given the low or high dose of either MLP or MLE orally, daily for $30 \mathrm{~d}$ and received a single intraperitoneal injection of CP ( 25 or $40 \mathrm{mg} / \mathrm{kg}$ body weight on day 25 or 29 , respectively) to induce immunosuppression ${ }^{(13,16)}$ or clastogenicity $^{(12)}$. The healthy control and CP-only-treated animals received $0.5 \mathrm{ml}$ distilled water orally and daily, as vehicle, instead of marjoram preparations for $30 \mathrm{~d}$.

\section{Blood and tissues sampling}

The animals were subjected to light diethyl ether anaesthesia before being killed on day 31. Blood was collected into clean test-tubes with or without EDTA. A portion of blood with EDTA was used for complete blood picture analysis by Coulter (Hemat 8 analyser; SEAC) and to estimate the activities of erythrocyte enzymes. Another blood portion without EDTA was used to separate serum, which was divided into samples and preserved at $-70{ }^{\circ} \mathrm{C}$ for further analysis. Immediately after killing the animals, the thymus and spleen were separated out of the body, cleaned and weighed. Bone marrow was also collected from the left femur bone. The cellularity of lymphoid organs was measured by a Neubauer counting chamber (Paul Marienfeld $\mathrm{GmbH}$ ) after lysing the erythrocytes using an erythrocyte lysis solution (AppliChem $\mathrm{GmbH})$. 


\section{Measurements}

Quantitative measurement of serum Ig concentration, as an indicator for humoral immune response, was performed using IgM and IgG pre-calibrated radial immunodiffusion plates (Biocientifica S.A.) according to the manufacturer's recommendations. Serum reduced and oxidised glutathione (GSH and GSSG, respectively) and malondialdehyde (MDA)

(A)
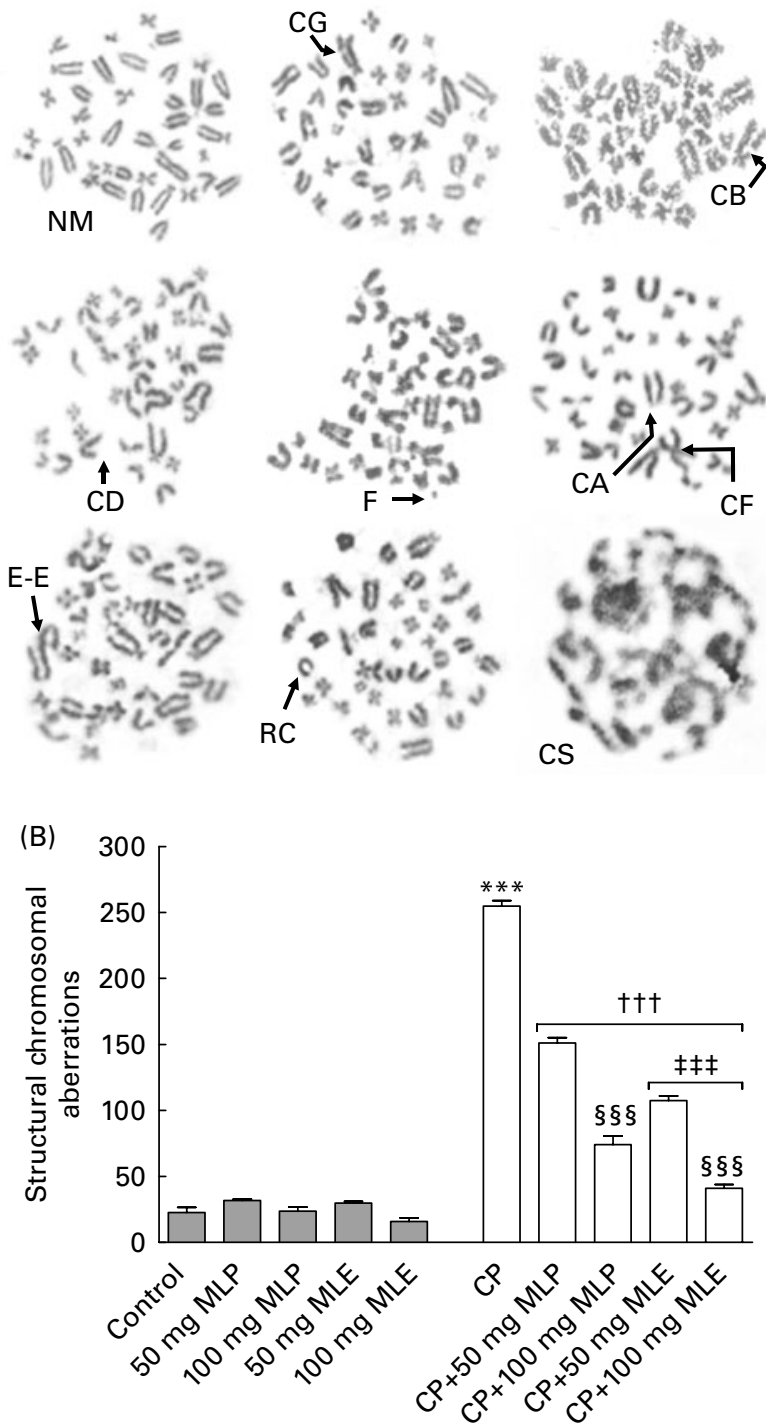

Fig. 1. (A) Different types (CA, centromeric attenuation; $C B$, chromatid break; $C D$, chromatid deletion; $C F$, centric fusion; CG, chromatid gap; $C S$ chromosome stickiness; E-E, end-to-end association; $F$, fragment; NM, normal metaphase; RC, ring chromosome) and (B) total count of structural chromosomal aberrations in bone marrow cells of cyclophosphamide (CP) and/or marjoram leaves-treated rats. Values are means, with their standard errors represented by vertical bars. ${ }^{* * *}$ Mean values were significantly different from that of the control group $(P<0.001)$. t†† Mean values were significantly different from that of the CP-only-treated group $(P<0.001)$. $\ddagger \ddagger \ddagger$ Mean values were significantly different from that of the CP-treated group that received the corresponding dose of marjoram leaf powder (MLP) suspension $(P<0.001)$. $\S \S \S M e a n$ values were significantly different from that of the CP-treated group that received $50 \mathrm{mg} / \mathrm{kg}$ body weight of the same marjoram preparation $(P<0.001)$. MLE, marjoram leaf aqueous extract. concentrations were measured by HPLC (HP1100 series; Agilent Technologies $)^{(24,25)}$. Total glutathione was calculated as follows: total glutathione $=\mathrm{GSH}+\mathrm{GSSG}$. Serum catalase (CAT) activity was estimated from the rate of formaldehyde formation in the presence of methanol and optimal concentration of $\mathrm{H}_{2} \mathrm{O}_{2}$. The produced formaldehyde was measured spectrophotometrically using 4-amino-3-hydrazino-5-mercapto-1,2,4-triazole as a chromogen ${ }^{(26)}$. Serum glutathione $S$ transferase (GST) activity was determined by measuring the conjugation rate of 1-chloro-2,4-dinitrobenzene with GSH, which is directly proportional to the GST activity ${ }^{(27)}$. Erythrocyte glutathione peroxidase (GPx; selenium-dependent) activity was indirectly determined by a coupled reaction with glutathione reductase. GSSG, produced upon reduction of an organic hydroperoxide by GPx, was recycled to its reduced state by glutathione reductase and NADPH. The rate of decrease in NADPH is directly proportional to the GPx activity ${ }^{(28)}$. Erythrocyte superoxide dismutase activity was determined by measuring the ability of the enzyme to inhibit the reduction of nitro-blue tetrazolium by superoxide, which was generated by the autoxidation of pyrogallol ${ }^{(29)}$. The percentage of change of any parameter $=((T-C) /$ $C) \times 100$, where $T=$ the mean value of the parameter in the treated group and $C=$ the mean value of the parameter in the control group.

\section{Chromosomal aberrations and mitotic index}

The animals received a single intraperitoneal injection of $2 \mathrm{~mm}$-colchicines $2 \mathrm{~h}$ before being killed to disrupt spindlefibre formation, prevent the migration of chromatids after splitting the centromere and generate diploid cells containing pairs of identical chromosomes that are homozygous at all loci ${ }^{(30)}$. Bone marrow cells were stained for chromosomal aberration analysis as described previously ${ }^{(30)}$. Briefly, bone marrow cells were incubated with $6 \mathrm{ml}$ of hypotonic $\mathrm{KCl}$ (0.65\%, Sigma-Aldrich) at $37^{\circ} \mathrm{C}$ for $20 \mathrm{~min}$. After centrifugation, the sediment was fixed three times in a mixture of methanol and acetic acid (3:1, Sigma-Aldrich) before slides preparation. Then, the slides were air-dried and stained with Giemsa solution (Sigma-Aldrich). Following this, 100 spread metaphases per animal were randomly examined for structural and numerical chromosomal aberrations using $100 \times$ oil immersion objective lenses. The mitotic index was also determined by calculating the percentage of cells in metaphase per 1000 cells for each animal.

\section{Delayed type of hypersensitivity response}

The delayed type of hypersensitivity response, as an indicator for cellular immune response, was determined as described previously $^{(16)}$ with some modifications. Briefly, on day 31 , the animals were subcutaneously immunised with $2 \cdot 25 \times 10^{10}$ sheep erythrocytes (Institute of the Agricultural Researches, Giza, Egypt). On the fifth day of immunisation, the animals were again challenged with $1 \times 10^{10}$ cells in the left hind footpad. The increase in footpad thickness was measured $24 \mathrm{~h}$ after the second challenge by a vernier calliper 
(Samir and Ali Bookshop, Cairo, Egypt). The right footpad was injected with saline solution and served as trauma control for non-specific swelling.

\section{Statistical analysis}

Data are presented as means with their standard errors. Statistical analysis was performed with one-way ANOVA, and the differences among groups were determined by Bonferroni's multiple comparison test ${ }^{(31)}$, using GraphPad Prism version 4.03 for Windows (GraphPad Software, Inc.). $P$ values of $<0 \cdot 05,<0 \cdot 01$ and $<0.001$ were considered statistically significant, highly significant and very highly significant, respectively.

\section{Results}

\section{Marioram leaves alleviated the genotoxicity induced by} cyclophosphamide in bone marrow cells of rats

The present study showed that treatment of rats with $\mathrm{CP}$ alone induced a very highly significant increase $(P<0.001$, $t=44.99$, difference between means $=-232 \cdot 2, \quad 95 \%$ CI $-248.9,-215.5)$ in nine of the structural chromosomal aberrations that were detected in bone marrow cells: chromatid gap (1733\%), chromatid break (1900\%), chromatid deletion (454\%), fragment (1450\%), centromeric attenuation (1500\%), centric fusion (2175\%), end-to-end association (1510\%), ring chromosomes (1525\%) and chromosome stickiness $(1485 \%)$, compared with the healthy control animals (Fig. 1). Also, it induced a very highly significant increase ( $P<0.001, t=13 \cdot 05$, difference between means $=-16 \cdot 40$, $95 \% \mathrm{CI}-20 \cdot 48,-12 \cdot 32)$ in three of the numerical chromosomal aberrations (aneuploidy) that were found in bone marrow cells of rats: monosomic $(2 n-1,1500 \%)$, trisomic $(2 n+1$, $2800 \%)$ and tetrasomic $(2 n+2,2400 \%)$, compared with the healthy control animals (Fig. 2(A)). On the other hand, $\mathrm{CP}$-only-treated rats showed a very highly significant decrease $(P<0.001, t=25.96$, difference between means $=9 \cdot 46,95 \%$ CI $8.28,10.64)$ in the mitotic index $(44 \%)$ of bone marrow cells, compared with the healthy control animals (Fig. 2(B)).

Both MLP and MLE significantly alleviated (14-100\%, $P<0.001$, compared with the CP-only-treated group) the increase in structural and numerical chromosomal aberrations and the decrease in mitotic index induced by $\mathrm{CP}$ in bone marrow cells of rats (Figs. 1 and 2). These modulatory effects significantly increased by increasing the dose of marjoram in all cases $(P<0 \cdot 01-0 \cdot 001)$, except in the mitotic index of bone marrow cells of CP-treated rats that received MLE where no statistically significant difference in modulation $(P>0.05)$ was found between both doses of MLE (Fig. 2(B)). The modulatory effects of MLE on the increase in the structural chromosomal aberrations (Fig. 1(B)) and the decrease in the mitotic index (Fig. 2(B)) in bone marrow cells of CP-treated rats were significantly higher $(10-17 \%, P<0.001)$ than that of MLP, especially at the low dose.

\section{Marjoram leaves alleviated the immunosuppression induced by cyclophosphamide in rats}

The present study showed that treatment of rats with CP alone induced severe blood leucopenia $(P<0.001, t=8.87$, difference between means $=3.99,95 \%$ CI $2.53,5.45)$, which was mainly due to a significant decrease $(P<0.001)$ in counts of all granulocytes (41-84\%) and agranulocytes (68-76\%), compared with the healthy control animals (Table 1). It also caused a significant decrease $(P<0.05-0.001)$ in serum IgM (51\%) and IgG (28\%) levels (data not shown) and the delayed type of hypersensitivity response (25\%) as well as weights (64-66\%) and cellularity (54-79\%) of lymphoid organs, compared with the healthy control animals (Figs. 3 and 4).
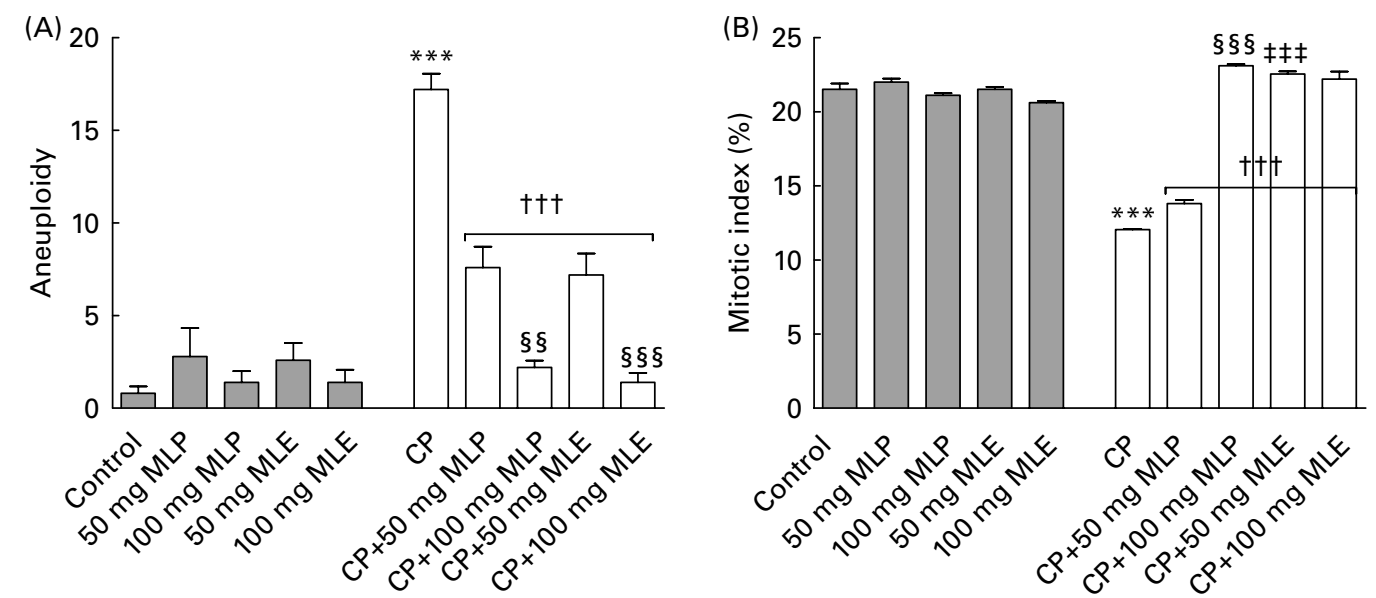

Fig. 2. (A) Aneuploidy numerical chromosomal aberrations and (B) mitotic index in bone marrow cells of cyclophosphamide (CP) and/or marjoram leaves-treated rats. Three types of aneuploidy (monosomic, trisomic and tetrasomic) were detected in the present study. Values are means, with their standard errors represented by vertical bars. ${ }^{* *}$ Mean values were significantly different from that of the control group $(P<0.001)$. $\dagger \dagger \dagger$ Mean values were significantly different from that of the CP-only-treated group $(P<0.001)$. $¥ \ddagger \ddagger$ Mean values were significantly different from that of the CP-treated group that received the corresponding dose of marjoram leaf powder (MLP) suspension $(P<0.001)$. Mean values were significantly different from that of the $\mathrm{CP}$-treated group that received $50 \mathrm{mg} / \mathrm{kg}$ body weight of the same marjoram preparation: $\S \S P<0.01$, §§§ $P<0.001$. MLE, marjoram leaf aqueous extract. 
Both MLP and MLE significantly alleviated $(P<0 \cdot 05-0 \cdot 001)$ all the immunosuppressive activity of $\mathrm{CP}$ including leucopenia (94-102\%, Table 1), the decrease in humoral (27-99\%, data not shown) and cellular (47-69\%) immune responses (Fig. 3) and the decrease in weights (76-160\%) and cellularity (74-593\%) of lymphoid organs (Fig. 4), except that the low dose of MLP did not significantly modulate $(P>0.05)$ the decrease in total granulocytes count (Table 1), the cellular immune response (Fig. 3) and bone marrow cellularity (Fig. 4(B)) in CP-plus-MLP-treated rats compared with CPonly-treated rats. Moreover, the delayed type of hypersensitivity response (Fig. 3) as well as the cellularity of thymus and spleen (Fig. 4(B)) in CP-treated animals that received the high dose of MLE exceeded that in the healthy control animals (27-44\%, P<0.05-0.001). The modulatory effects on the cellularity of spleen and bone marrow significantly increased $(P<0.01$ and $P<0 \cdot 001$, respectively) by increasing the dose of MLP (Fig. 4(B)). In addition, the modulatory effects on total agranulocytes count (Table 1 ) and the cellularity of lymphoid organs (Fig. 4(B)) significantly increased $(P<0.05-$ $0.001)$ by increasing the dose of MLE. The modulatory effects of MLE on the decrease in the cellularity of bone marrow and spleen in CP-treated rats were significantly higher (132-172\%, $P<0 \cdot 001$ ) than that of MLP (Fig. 4(B)).

\section{Marioram leaves alleviated the haematotoxicity induced by cyclophosphamide in rats}

The present study showed that treatment of rats with $\mathrm{CP}$ alone induced a highly significant decrease (9-35\%,P<0.01-0.001) in erythrocyte and platelet counts and the $\mathrm{Hb}$ content and a highly significant increase $(18 \%, P<0.01-0.001)$ in the mean corpuscular volume and mean corpuscular Hb (Fig. 5), but did not significantly change $(1-9 \%, P>0.05)$ the haematocrit value and mean corpuscular $\mathrm{Hb}$ concentration (data not shown), compared with the healthy control animals. Both MLP and MLE significantly alleviated (12-60\%, $P<0.05-$ $0 \cdot 001$, compared with CP-only-treated rats) the macrocytic normochromic anaemia and thrombocytopenia caused by $\mathrm{CP}$ in rats (Fig. 5). These modulatory effects did not significantly change $(P>0.05)$ by increasing the dose of either MLP or MLE. In addition, there was no significant difference $(P>0.05)$ between the modulatory effects of MLP and MLE on the haematotoxicity induced by $\mathrm{CP}$ in rats (Fig. 5).

\section{Marioram leaves alleviated the oxidative stress induced by cyclophosphamide in rats}

The present study showed that treatment of rats with $\mathrm{CP}$ alone induced a very highly significant increase in serum MDA level ( $43 \%, P<0.001, t=5.96$, difference between means $=-0 \cdot 14,95 \% \mathrm{CI}-0 \cdot 22,-0.06)$ and a significant decrease in the level of serum non-enzymic antioxidants (26-65\%, P<0.05-0.001) and the activity of serum and erythrocyte enzymic antioxidants $(11-46 \%, P<0 \cdot 001)$, compared with the healthy control animals (Table 2). Low dose of MLP significantly alleviated (20-78\%, P<0.01-0.001, compared with $\mathrm{CP}$-only-treated rats) only the decrease in the activity 


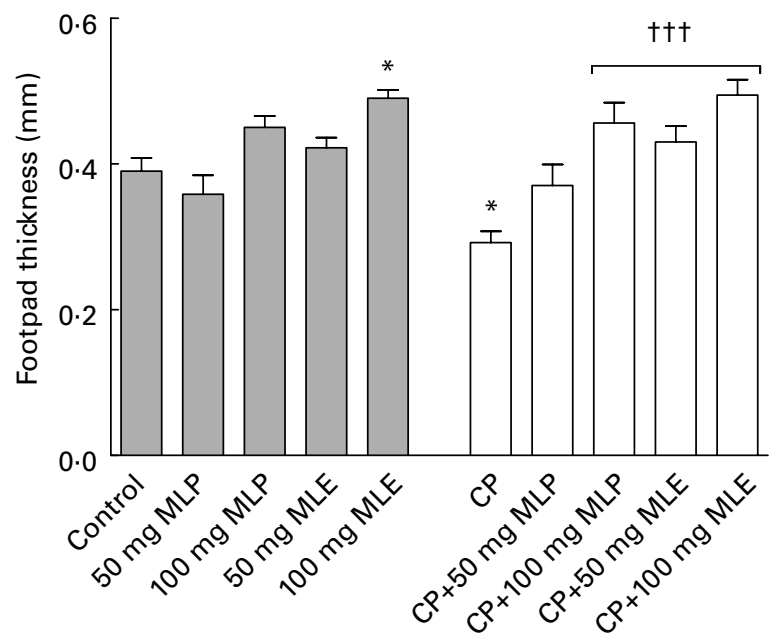

Fig. 3. Delayed type of hypersensitivity response of cyclophosphamide (CP) and/or marjoram leaves-treated rats. Values are means, with their standard errors represented by vertical bars. * Mean values were significantly different from that of the control group $(P<0.05)$. $+\dagger \dagger$ Mean values were significantly different from that of the CP-only-treated group $(P<0.001)$. MLE, marjoram leaf aqueous extract; MLP, marjoram leaf powder.

of serum CAT and GST induced by CP in rats. The high dose of MLP significantly alleviated (17-86\%, P<0.05-0.001, compared with $\mathrm{CP}$-only-treated rats) the increase in serum MDA level as well as the decrease in serum total glutathione level and the activity of serum CAT and GST in CP-treated rats (Table 2). On the other hand, both doses of MLE significantly alleviated (9-184\%, P<0.05-0.001, compared with CP-onlytreated rats) the increase in the cellular toxicity and the decrease in the level/activity of all non-enzymic/enzymic antioxidants measured in serum and erythrocytes of animals that received $\mathrm{CP}$ (Table 2 ). These modulatory effects did not significantly change $(P>0.05)$ by increasing the dose of either MLP or MLE. On the other hand, the modulatory effects of MLE on the increase in the cellular toxicity and the decrease in the activity of erythrocyte GPx were significantly higher (33-39\%, $P<0.05-P<0.001)$ than that of MLP, especially at the high dose (Table 2).

The percentages of changes of all parameters measured in the CP-only-treated group, the groups treated with $\mathrm{CP}$ plus either the low or high dose of MLP, and the groups treated with CP plus either the low or high dose of MLE were 453.5 (SEM 116.4, $P<0 \cdot 001$ ), $216 \cdot 3$ (sem 53.8, $P<0 \cdot 01$ ), 74.4 (sem $24.3, P>0.05$ ), 162.2 (SEM $41.0, P>0.05$ ) and 29.9 (SEM 11.5 , $P>0.05$ ), respectively, compared with the healthy control group. All of these results revealed that the utmost modulation on the serious side effects detected in the present study in CP-treated rats was induced by the high dose of MLE.

\section{Beneficial and deleterious effects caused by marjoram leaves consumption in healthy rats}

The present study showed that treatment of healthy rats with high dose of either MLP or MLE alone significantly increased $(P<0.05-0.001)$ the cellularity of thymus and spleen (22-45\%, Fig. 4(B)), serum total glutathione level, GSH:GSSG ratio and GST activity (27-160\%, Table 2$)$. In addition, the low and high dose of MLE significantly increased the activity of serum CAT (23-52\%, $P<0.001)$ and erythrocyte superoxide dismutase $(8-9 \%, P<0.05)$ in healthy animals (Table 2).
(A)

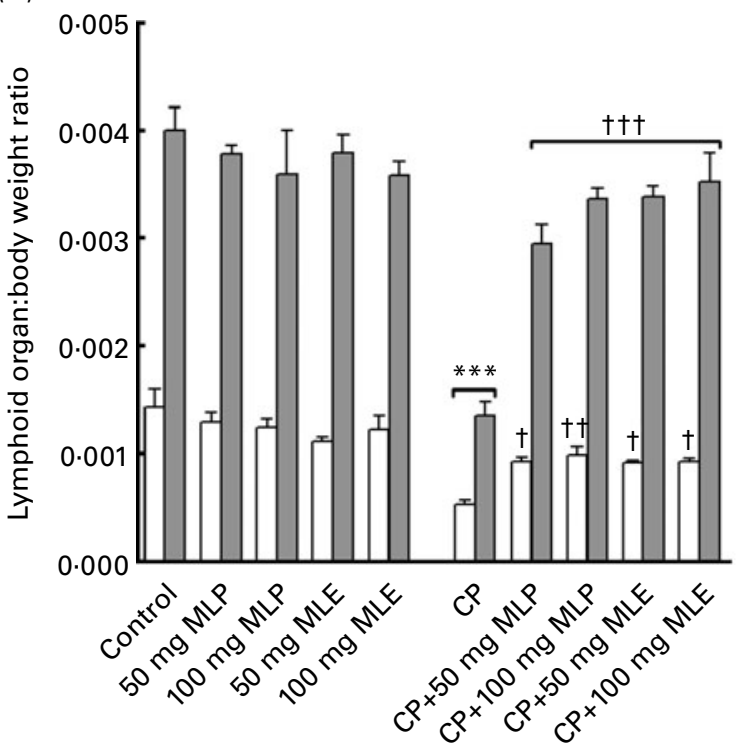

(B)

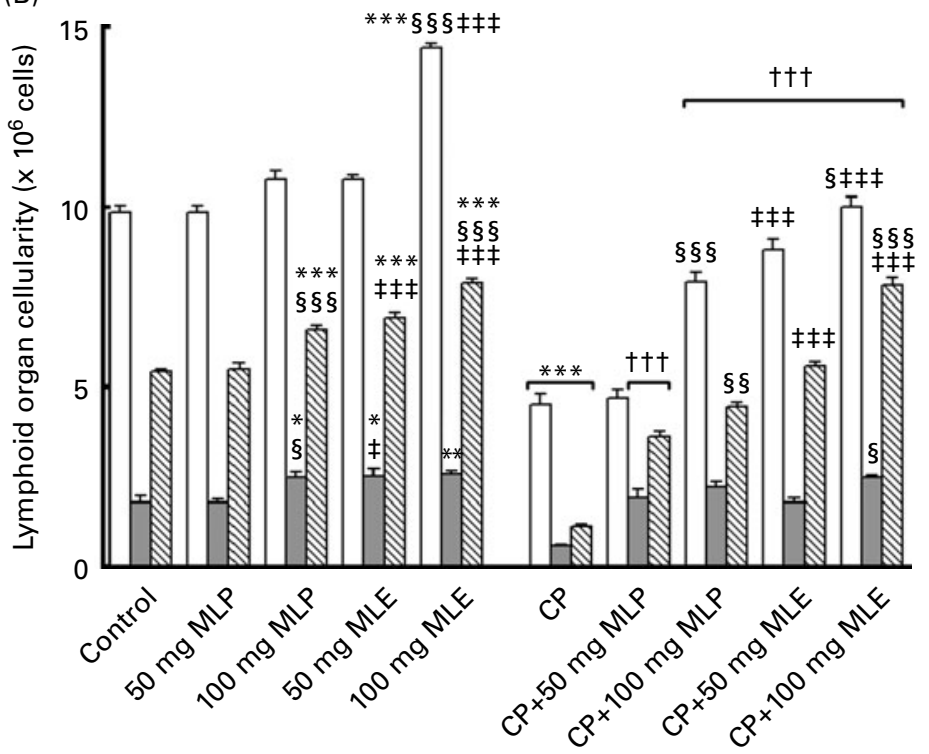

Fig. 4. (A) Weight ( $\square$, thymus; $\square$, spleen) and (B) cellularity ( $\square$, bone marrow; $\square$, thymus; 四, spleen) of lymphoid organs of cyclophosphamide (CP) and/or marjoram leaves-treated rats. Values are means, with their standard errors represented by vertical bars. Mean values were significantly different from that of the control group: ${ }^{*} P<0.05,{ }^{* *} P<0.01,{ }^{* * *} P<0.001$. Mean values were significantly different from that of the CP-only-treated group: $\dagger P<0.05, \dagger \dagger P<0.01$, t†† $P<0.001$. Mean values were significantly different from that of the CP-treated group that received the corresponding dose of marjoram leaf powder (MLP) suspension: $\ddagger P<0.05$, 㧊 $P<0.001$. Mean values were significantly different from that of the CP-treated group that received $50 \mathrm{mg} / \mathrm{kg}$ body weight of the same marjoram preparation: $\S P<0.05, \S \S P<0.01, \S \S \S P<0.001$. MLE, marjoram leaf aqueous extract. 
(A)

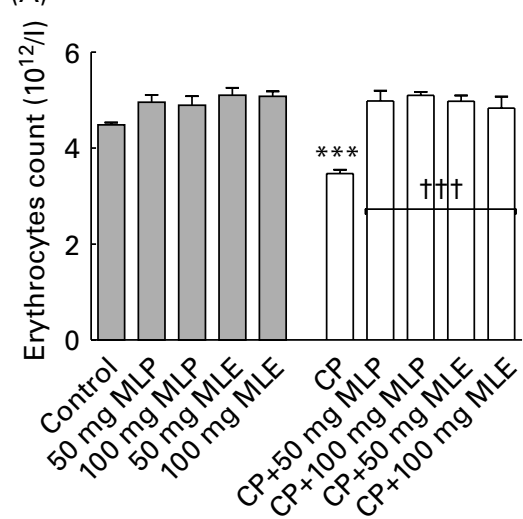

(C)

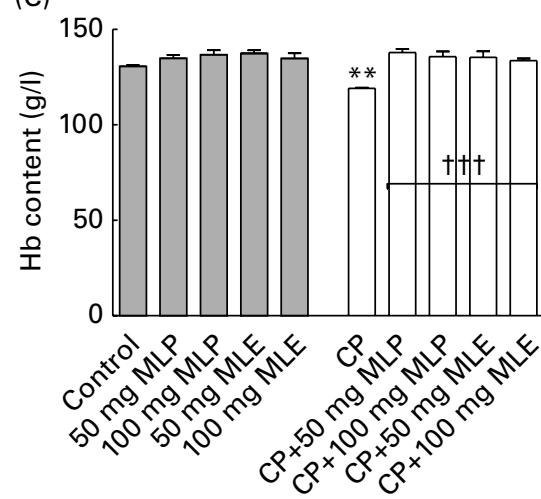

(B)

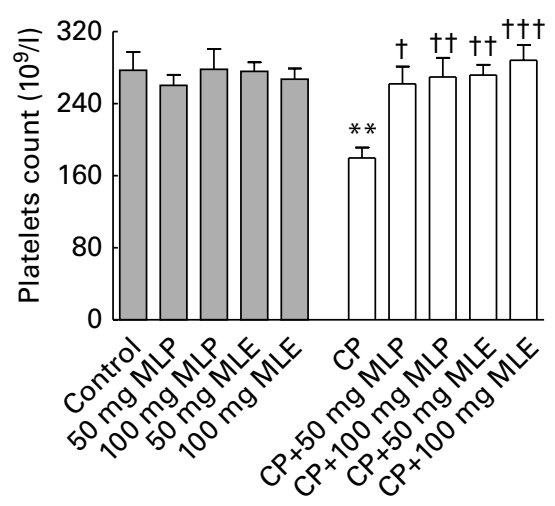

(D)

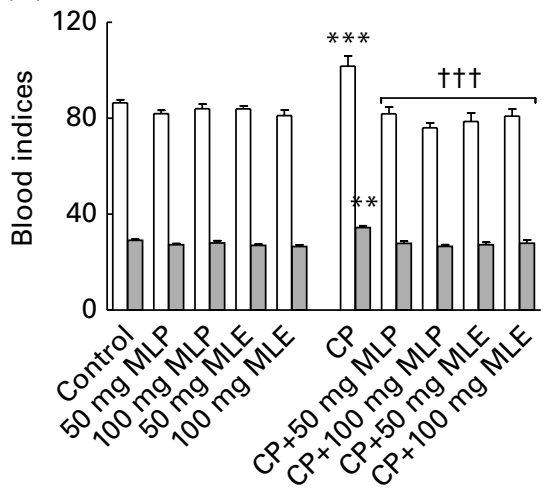

Fig. 5. (A) Erythrocytes count, (B) platelets count, (C) Hb content and (D) blood indices ( $\square$, mean corpuscular volume (fl); $\square$, mean corpuscular $\mathrm{Hb}$ (MCH, pg)) of cyclophosphamide (CP) and/or marjoram leaves-treated rats. The haematocrit value and $\mathrm{MCH}$ concentration did not significantly change among all groups. Values are means, with their standard errors represented by vertical bars. Mean values were significantly different from that of the control group: ${ }^{\star \star} P<0.01,{ }^{* \star \star} P<0.001$. Mean values were significantly different from that of the CP-only-treated group: $\uparrow P<0.05, \dagger \dagger P<0.01, \dagger \dagger \uparrow<0.001$. MLE, marjoram leaf aqueous extract; MLP, marjoram leaf powder.

Moreover, the low dose of MLE significantly increased the cellularity of thymus and spleen (28-41\%, P<0.05-0.001, Fig. 4(B)), while the high dose of MLE significantly increased the delayed type of hypersensitivity response $(26 \%, P<0 \cdot 05$, Fig. 3), bone marrow cellularity ( $46 \%, P<0 \cdot 001$, Fig. 4(B)) and erythrocyte GPx activity $(20 \%, P<0.05$, Table 2$)$ in healthy animals. These beneficial effects significantly increased by increasing the dose of marjoram $(P<0.05-0.001)$ and were significantly higher in MLE $(P<0.05-0.001)$ in most cases. All other parameters measured in the present study did not significantly alter in healthy rats by both doses of either MLP or MLE $(P>0.05)$. Moreover, food intake, body weight, blood total and differential leucocyte counts, haematocrit value and mean corpuscular $\mathrm{Hb}$ concentration were not significantly changed $(P>0.05)$ in healthy rats that received either MLP or MLE, compared with the healthy control animals (data not shown). All of these results indicated that the marjoram doses used in the present study were considered safe.

\section{Discussion}

$\mathrm{CP}$ itself is an inactive cytostatic devoid of alkylating activity and must first undergo metabolic activation catalysed by the hepatic cytochrome P450 monoxygenase systems ${ }^{(1,2,11,32)}$. Among the CP-reactive metabolites, phosphoramide mustard and acrolein are specially associated with the immunosuppressive action and oxygen radical formation of $\mathrm{CP}$, respectively ${ }^{(3,14,32)}$. Acrolein also deprives the cells of its natural defence (GSH) against reactive oxygen species by interacting with its amino acid, cysteine ${ }^{(33)}$. Free radicals exert their toxic effects by acting on cellular macromolecules as DNA, membrane proteins and lipids ${ }^{(15)}$. The genotoxicity and chromosomal instability induced by many clastogenic agents are straight correlated with their ability to increase the oxidative stress $^{(34-36)}$. Therefore, the oxidative stress arising from the overproduction of reactive oxygen species and the weakness in the antioxidant defence system by CP metabolites is involved in the pathogenesis of most of the side effects of CP.

The present study showed (probably for the first time) that both MLP and MLE (especially the high dose) significantly alleviated $(P<0.05-0.001)$ most of the side effects and toxicity of $\mathrm{CP}$-treated rats including the increase in the structural and numerical chromosomal aberrations of bone marrow cells and serum MDA level, the deficiency in humoral and cellular immune responses and myelosuppression. The increase in the weight of lymphoid organs (thymus and spleen) induced by 
MLP and MLE in CP-treated animals was concomitant with the increase in their cellularity (Fig. 4). The modulatory effects of MLP and MLE on CP-induced myelosuppression were probably due to their ability to increase the mitotic index of bone marrow cells as shown in Fig. 2(B). The anticlastogenic and immunostimulatory activities of MLP and MLE shown in the present study may reduce the incidence of secondary tumours and infections, respectively, in cancer patients treated with $\mathrm{CP}$ or other chemotherapeutic agents. Other studies found that sweet marjoram extract and its component carvacrol induced apoptosis (programmed cell death) and showed antitumour activity against a number of human cancer cell lines and mouse N-ras transformed myoblast cells ${ }^{(37-39)}$. On the other hand, the antimicrobial activity of marjoram was attributed to ursolic acid, thymol and carvacrol $^{(17,40)}$.

As shown in the present study (Table 2), one of the possible mechanisms for the beneficial effects of MLP and MLE in CP-treated animals was their ability to reduce CP-induced oxidative stress (as indicated by the decrease in the level of MDA, an indicator of lipid peroxidation and membrane damage) through reactivating the non-enzymic (GSH) and enzymic (CAT, GPx, GST, superoxide dismutase) antioxidant system. MDA can also react with deoxyadenosine and deoxyguanosine in DNA and form DNA adducts that are mutagenic $^{(41)}$. A number of GSH-inducing compounds have been found to be effective in reducing CP toxicity in animals $^{(2,3,10,11,14,16,42)}$. In addition, the toxic metabolites of $\mathrm{CP}$, which are responsible for the drug urotoxicity, are mainly detoxified by $\mathrm{GST}^{(43)}$. Two previous studies also reported that the protective activity of sweet marjoram extracts and volatile oil against the clastogenic agents, hydroquinone and lead acetate, was mainly due to its ability to scavenge the free radicals ${ }^{(17,18)}$. One of these two studies ${ }^{(18)}$ did not investigate the antioxidant activity of sweet marjoram. In addition, the protective effect of sweet marjoram on the chromosomal aberrations induced by hydroquinone in bone marrow cells was weak, because the clastogenic activity of the hydroquinone itself was weak in the aforementioned study $^{(18)}$. The antioxidant activity of sweet marjoram was due to its high content of carnosol, carnosic acid, carvacrol,

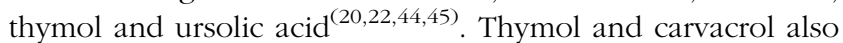
reduced the oxidative DNA damage in human lymphocytes $^{(44)}$. Another compound, T3b, which was isolated from the methanol extract of sweet marjoram, showed strong antioxidant properties and may possess anticancer activity ${ }^{(21)}$. Its activity was approximately five times greater than that of $\alpha$-tocopherol, an efficient natural $\mathrm{O}_{2}^{-}$scavenger $^{(21)}$. Because free radicals are responsible for the pathogenesis of $\mathrm{CP}$ induced dyslipidaemia, which is a well-known risk factor for CVD $^{(16,46)}$, the antioxidant activity of sweet marjoram shown in the present study may also protect against hyperlipidaemic cardiomyopathy in CP-treated cancer patients. The anticlastogenic and immunostimulatory activities of sweet marjoram may also be attributed to its ability for DNA repair and mitogenic stimulation of the immunocompetent cells, respectively.

In the present study, it was not determined whether the protection offered by the sweet marjoram leaves was related 
to a reduction in the efficacy of $\mathrm{CP}$ (by affecting the pharmacokinetics of this compound) or to a direct effect on the cells themselves. This topic needs further investigation and will be considered in the future. However, previous studies reported that an antioxidant dietary supplement can prevent the oxidation of biomolecules, including DNA, without decreasing the effectiveness of chemotherapeutic agents ${ }^{(13,15,47,48)}$. It was also reported that therapy with antioxidants concomitant with chemotherapy reduces the frequency and severity of side effects associated with many drugs in cancer patients, thereby allowing the treatment to be continued, since the toxicity of anticancer drugs is a frequent limitation to their extended $u^{(49)}$. Many plants were found to be effective in reducing or eliminating some of the undesirable side effects of $\mathrm{CP}$ in different animal models ${ }^{(2-4,7,10-16)}$. Interestingly, Egyptian sweet marjoram leaves were found to be effective in the present study against most of the side effects caused by CP including genotoxicity, myelosuppression, immunosuppression and oxidative stress. The modulatory effects of marjoram leaves shown in the present study were dose dependent in most cases and much higher in MLE (21-23\% for all parameters taken together). Moreover, the high dose of MLE showed stronger immunostimulatory activity in healthy animals (Figs. 3 and 4(B)). The superior modulatory effects of MLE (especially the high dose) shown in the present study were probably due to its superior beneficial effect on the non-enzymic and enzymic antioxidant system (Table 2). No harmful effects were detected for sweet marjoram leaves on any of the parameters measured in the present study, indicating that the doses used in this study were considered safe. In general, people in different countries consume about $2.5-5 \mathrm{~g} /$ $\mathrm{d}$ of sweet marjoram leaves in culinary use, which was 4-5 times more than the dose used in the present study for animals. In conclusion, sweet marjoram leaves (especially in the form of a herbal tea) may be useful as an immunostimulant and in reducing genotoxicity in patients under chemotherapeutic interventions.

\section{Acknowledgements}

This research received no specific grant from any funding agency in the public, commercial or not-for-profit sectors. The authors thank Nadia M. S. Arafa (Department of Physiology, National Organization for Drug Control and Research, Giza, Egypt) for technical help/support in HPLC analysis. N. M. E.-B. and G. R. planned the study, designed all experiments, summarised, discussed and interpreted the results, and drafted the manuscript. M. M. Z. carried out the experiments and performed the statistical analysis with assistance from G. R. The authors have no potential financial conflict of interest.

\section{References}

1. McCarroll N, Keshava N, Cimino M, et al. (2008) An evaluation of the mode of action framework for mutagenic carcinogens case study: cyclophosphamide. Environ Mol Mutagen 49, 117-131.
2. Haque R, Bin-Hafeez B, Parvez S, et al. (2003) Aqueous extract of walnut (Juglans regia L.) protects mice against cyclophosphamide-induced biochemical toxicity. Hum Exp Toxicol 22, 473-480.

3. Bhatia K, Kaur M, Atif F, et al. (2006) Aqueous extract of Trigonella foenum-graecum L. ameliorates additive urotoxicity of buthionine sulfoximine and cyclophosphamide in mice. Food Chem Toxicol 44, 1744-1750.

4. Arafa HM (2009) Uroprotective effects of curcumin in cyclophosphamide-induced haemorrhagic cystitis paradigm. Basic Clin Pharmacol Toxicol 104, 393-399.

5. Anderson D, Bishop JB, Garner RC, et al. (1995) Cyclophosphamide: review of its mutagenicity for an assessment of potential germ cell risks. Mutat Res 330, 115-181.

6. Burgaz S, Karahalil B, Bayrak P, et al. (1999) Urinary cyclophosphamide excretion and micronuclei frequencies in peripheral lymphocytes and in exfoliated buccal epithelial cells of nurses handling antineoplastics. Mutat Res 439, 97-104.

7. Bin-Hafeez B, Ahmad I, Haque R, et al. (2001) Protective effect of Cassia occidentalis L. on cyclophosphamideinduced suppression of humoral immunity in mice. J Ethnopharmacol 75, 13-18.

8. Zandvoort A, Lodewijk ME, Klok PA, et al. (2001) Slow recovery of follicular $\mathrm{B}$ cells and marginal zone $\mathrm{B}$ cells after chemotherapy: implications for humoral immunity. Clin Exp Immunol 124, 172-179.

9. Angulo I, Jimenez-Diaz MB, Garcia-Bustos JF, et al. (2002) Candida albicans infection enhances immunosuppression induced by cyclophosphamide by selective priming of suppressive myeloid progenitors for NO production. Cell Immunol 218, 46-58.

10. Sharma N, Trikha P, Athar M, et al. (2000) Inhibitory effect of Emblica officinalis on the in vivo clastogenicity of benzo[a]pyrene and cyclophosphamide in mice. Hum Exp Toxicol 19, 377-384.

11. Haque R, Bin-Hafeez B, Ahmad I, et al. (2001) Protective effects of Emblica officinalis Gaertn. in cyclophosphamidetreated mice. Hum Exp Toxicol 20, 643-650.

12. Shukla Y, Arora A \& Taneja P (2002) Antimutagenic potential of curcumin on chromosomal aberrations in Wistar rats. Mutat Res 515, 197-202.

13. Ramadan G, El-Beih NM \& Badr GM (2006) Modulatory effects of aged garlic extract in comparison with fresh garlic extract: II - On induced immunosuppression and systemic anaphylaxis in male albino rats. Egypt J Zool $\mathbf{4 6}$, 265-283.

14. Al-Yahya AA, Al-Majed AA, Gado AM, et al. (2009) Acacia senegal gum exudate offers protection against cyclophosphamide-induced urinary bladder cytotoxicity. Oxid Med Cell Longev 2, 207-213.

15. Popov B, Georgieva Sv \& Gadjeva V (2011) Modulatory effects of total extract of Haberlea rhodopensis against the cyclophosphamide induced genotoxicity in rabbit lymphocytes in vivo. Trakia J Sci 9, 51-57.

16. Ramadan G, El-Beih NM \& Abd El-Kareem HF (2011) Anti-metabolic syndrome and immunostimulant activities of Egyptian fenugreek seeds in diabetic/obese and immunosuppressive rat models. Br J Nutr 105, 995-1004.

17. El-Ashmawy IM, El-Nahas AF \& Salama OM (2005) Protective effect of volatile oil, alcoholic and aqueous extracts of Origanum majorana on lead acetate toxicity in mice. Basic Clin Pharmacol Toxicol 97, 238-243.

18. Ghaly IS, Said A \& Abdel-Wahhab MA (2008) Zizyphus jujuba and Origanum majorana extracts protect against hydroquinone-induced clastogenicity. Environ Toxicol Pharmacol 25, 10-19. 
19. Novak J, Bitsch C, Langbehn J, et al. (2000) Ratios of cis- and trans-sabinene hydrate in Origanum majorana L. and Origanum microphyllum (Bentham) vogel. Biochem Syst Ecol 28, 697-704.

20. Vagi E, Rapavi E, Hadolin M, et al. (2005) Phenolic and triterpenoid antioxidants from Origanum majorana L. herb and extracts obtained with different solvents. J Agric Food Chem 53, 17-21.

21. Jun WJ, Han BK, Yu KW, et al. (2001) Antioxidant effects of Origanum majorana L. on superoxide anion radicals. Food Chem 75, 439-444.

22. Heo HJ, Cho HY, Hong B, et al. (2002) Ursolic acid of Origanum majorana L. reduces Abeta-induced oxidative injury. Mol Cells 13, 5-11.

23. Ramadan G, El-Beih NM \& Abd El-Ghffar EA (2009) Modulatory effects of black $v$. green tea aqueous extract on hyperglycaemia, hyperlipidaemia and liver dysfunction in diabetic and obese rat models. Br J Nutr 102, 1611-1619.

24. Jayatilleke E \& Shaw S (1993) A high-performance liquid chromatographic assay for reduced and oxidized glutathione in biological samples. Anal Biochem 214, 452-457.

25. Karatepe M (2004) Simultaneous determination of ascorbic acid and free malondialdhyde in human serum by HPLCUV. LC GC N Am 22, 362-365.

26. Johansson LH \& Borg LA (1988) A spectrophotometric method for determination of catalase activity in small tissue samples. Anal Biochem 174, 331-336.

27. Habig WH, Pabst MJ \& Jakoby WB (1974) Glutathione $S$-transferases. The first enzymatic step in mercapturic acid formation. J Biol Chem 249, 7130-7139.

28. Paglia DE \& Valentine WN (1967) Studies on the quantitative and qualitative characterization of erythrocyte glutathione peroxidase. J Lab Clin Med 70, 158-169.

29. Minami M \& Yoshikawa H (1979) A simplified assay method of superoxide dismutase activity for clinical use. Clin Chim Acta 92, 337-342.

30. El-Beih NM, Ramadan G \& Badr GM (2006) Modulatory effects of aged garlic extract in comparison with fresh garlic extract: I - On induced liver toxicity and genotoxicity in male albino rats. Egypt J Zool 46, 245-264.

31. Turner JR \& Thayer JF (editors) (2001) Introduction to Analysis of Variance: Design, Analysis and Interpretation. Thousand Oaks, CA: Sage Publications.

32. Boddy AV \& Yule SM (2000) Metabolism and pharmacokinetics of oxazaphosphorines. Clin Pharmacokinet $\mathbf{3 8}$, 291-304

33. Kehrer JP \& Biswal SS (2000) The molecular effects of acrolein. Toxicol Sci 57, 6-15.

34. Hussain SP, Hofseth LJ \& Harris CC (2003) Radical causes of cancer. Nat Rev Cancer 3, 276-285.
35. Jahangir T, Khan TH, Prasad L, et al. (2005) Pluchea lanceolata attenuates cadmium chloride induced oxidative stress and genotoxicity in Swiss albino mice. J Pharm Pharmacol 57, 1199-1204

36. Lee DH, Lim BS, Lee YK, et al. (2006) Involvement of oxidative stress in mutagenicity and apoptosis caused by dental resin monomers in cell cultures. Dent Mater 22, 1086-1092

37. Lin LT, Liu LT, Chiang LC, et al. (2002) In vitro anti-hepatoma activity of fifteen natural medicines from Canada. Phytother Res 16, 440-444.

38. Zeytinoglu H, Incesu $\mathrm{Z}$ \& Baser KH (2003) Inhibition of DNA synthesis by carvacrol in mouse myoblast cells bearing a human N-RAS oncogene. Phytomedicine 10, 292-299.

39. Arunasree KM (2010) Anti-proliferative effects of carvacrol on a human metastatic breast cancer cell line, MDA-MB 231. Phytomedicine 17, 581-588.

40. Leeja L \& Thoppil JE (2007) Antimicrobial activity of methanol extract of Origanum majorana L. (Sweet marjoram). J Environ Biol 28, 145-146.

41. Li Z, Henning SM, Zhang Y, et al. (2010) Antioxidant-rich spice added to hamburger meat during cooking results in reduced meat, plasma, and urine malondialdehyde concentrations. Am J Clin Nutr 91, 1180-1184.

42. Manesh C \& Kuttan G (2005) Effect of naturally occurring isothiocyanates in the inhibition of cyclophosphamideinduced urotoxicity. Phytomedicine 12, 487-493.

43. Zhong S, Huang M, Yang X, et al. (2006) Relationship of glutathione $S$-transferase genotypes with side-effects of pulsed cyclophosphamide therapy in patients with systemic lupus erythematosus. Br J Clin Pharmacol 62, 457-472.

44. Aydin S, Basaran AA \& Basaran N (2005) Modulating effects of thyme and its major ingredients on oxidative DNA damage in human lymphocytes. J Agric Food Chem 53, $1299-1305$.

45. Hazzit M, Baaliouamer A, Faleiro ML, et al. (2006) Composition of the essential oils of Thymus and Origanum species from Algeria and their antioxidant and antimicrobial activities. J Agric Food Chem 54, 6314-6321.

46. Mythili Y, Sudharsan PT, Sudhahar V, et al. (2006) Protective effect of DL-alpha-lipoic acid on cyclophosphamide induced hyperlipidemic cardiomyopathy. Eur J Pharmacol 543, $92-96$.

47. Liu X, Zhao J \& Zheng R (2003) DNA damage of tumorassociated lymphocytes and total antioxidant capacity in cancerous patients. Mutat Res 539, 1-8.

48. Berger MM (2005) Can oxidative damage be treated nutritionally? Clin Nutr 24, 172-183.

49. Borek C (2004) Dietary antioxidants and human cancer. Integr Cancer Ther 3, 333-341. 